\title{
Homologous Regulation of Mu Opioid Receptor Recycling by $\mathrm{G}_{\beta \gamma}$, Protein Kinase $\mathrm{C}$, and Receptor Phosphorylation $\$$
}

\author{
(D) Jennifer M. Kunselman, Amanda S. Zajac, ${ }^{1}$ @Zara Y. Weinberg, \\ and () Manojkumar A. Puthenveedu \\ Cellular and Molecular Biology Program (J.M.K., M.A.P.) and Department of Pharmacology (J.M.K., Z.Y.W., M.A.P.), University of \\ Michigan, Ann Arbor, Michigan; and Department of Biological Sciences, Carnegie Mellon University, Pittsburgh, Pennsylvania \\ (A.S.Z., M.A.P.)
}

Received May 17, 2019; accepted September 14, 2019

\section{ABSTRACT}

Membrane trafficking and receptor signaling are two fundamental cellular processes that interact constantly. Although how trafficking regulates signaling is well studied, how signaling pathways regulate trafficking is less well understood. Here, we use the mu opioid receptor (MOR), the primary target for opioid analgesics, to define a signaling pathway that dynamically regulates postendocytic receptor recycling. By directly visualizing individual MOR recycling events, we show that agonist increases MOR recycling. Inhibition of $\mathrm{G}_{\beta \gamma}$, phospholipase $\mathrm{C}$, or protein kinase $\mathrm{C}$ mimicked agonist removal, whereas activation of $\mathrm{G}_{\beta \gamma}$ increased recycling even after agonist removal. Phosphorylation of serine 363 on the C-terminal tail of MOR was required and sufficient for agonist-mediated regulation of MOR recycling. Our results identify a feedback loop that regulates MOR recycling via $\mathrm{G}_{\beta \gamma}$, protein kinase $\mathrm{C}$, and receptor phosphorylation. This could serve as a general model for how signaling regulates postendocytic trafficking of $G$ protein-coupled receptors.

\section{SIGNIFICANCE STATEMENT}

G protein-coupled receptor (GPCR) localization in the endosome is being increasingly recognized as an important and distinct component of GPCR signaling and physiology. This study identifies a $G$ protein-dependent and protein kinase C-dependent signaling pathway that dynamically regulates the endosomal localization of the mu opioid receptor, the primary target of opioid analgesics and abused drugs. This pathway could provide a mechanism to manipulate spatial encoding of opioid signaling and physiology.

\section{Introduction}

The dynamic relationship between trafficking and signaling of receptors is currently being redefined by new paradigms that are emerging in the field. This is especially true in the case of G protein-coupled receptors (GPCRs), the largest class of signaling receptors in humans (Pierce et al., 2002; Sriram and Insel, 2018). Activated GPCRs signal via multiple signaling pathways, some of which cause receptors to be internalized and trafficked to endosomes. Internalized GPCRs may be

M.A.P. was supported by the National Institutes of Health (NIH) National Institute of General Medical Sciences [Grant R01 GM117425] and the National Science Foundation (NSF) [Grant 1517776]. J.M.K. was supported by the NIH National Institute of General Medical Sciences [Grant T-32 GM007315].

The authors have no competing interests to report.

${ }^{1}$ Current affiliation: Division of Infectious Diseases, Department of Medicine, Massachusetts General Hospital, Boston, Massachusetts 02114 USA; Department of Microbiology, Blavatnik Institute, Harvard Medical School, Boston, Massachusetts 02115, USA.

Data and materials availability: There are no proprietary reagents or data used in this paper. Data will be made freely available on request subject to University of Michigan regulations and agreements.

https://doi.org/10.1124/mol.119.117267.

S This article has supplemental material available at molpharm. aspetjournals.org. either recycled back to the surface or degraded. Classically, this endocytic trafficking was thought to primarily control the desensitization and resensitization of signaling via $G$ proteins by removing receptors from or returning receptors to the cell surface (Lefkowitz et al., 1997; Marchese et al., 2008). However, recent evidence shows that many GPCRs can signal from intracellular sites, and that intracellular signaling can have distinct downstream effects compared with signaling from the surface (Irannejad et al., 2013; Vilardaga et al., 2014; Thomsen et al., 2018). Intracellular signaling from endosomal microdomains might be a general characteristic of GPCRs, as the list of GPCRs that can signal from intracellular compartments is still growing (Caengprasath and Hanyaloglu, 2019; Weinberg et al., 2019). This supports the emerging idea that an equally important role of trafficking is to transfer receptors between distinct signaling environments within a cell.

In the endosome, receptors need to be localized to specific endosomal microdomains both to sort into specific recycling pathways and to signal (Ferrandon et al., 2009; Vistein and Puthenveedu, 2013; Bowman et al., 2016). The localization of receptors in these domains and their further intracellular sorting are mediated by specific sequences on the C-terminal

ABBREVIATIONS: $\beta 2 A R, \beta 2$-adrenoreceptor; DAMGO, [D-Ala2, N-MePhe4, Gly-ol]-enkephalin; GPCR, G protein-coupled receptor; MOR, mu opioid receptor; mSIRK, myr-SIRKALNILGYPDYD-OH; PDZ, post-synaptic density-95/disc large tumor suppressor/zonula occludens-1; PKA, protein kinase A; PKC, protein kinase C; PLC, phospholipase C; PTX, pertussis toxin; SpH, superecliptic phluorin; WT, wild-type. 
tails of the receptors (Tanowitz and von Zastrow, 2003; Bowman and Puthenveedu, 2015; Bahouth and Nooh, 2017). For some of these sequences, interacting proteins that mediate this sorting and recycling have been identified (Romero et al., 2011; Dunn and Ferguson, 2015; Broadbent et al., 2017). Nevertheless, how this sorting and recycling is dynamically regulated by signaling pathways is a fundamental question that is still not well understood.

The mu opioid receptor (MOR) is an excellent model GPCR that can be used to address this question. MOR is highly physiologically relevant, as it is the primary target for many currently used and abused opioid drugs. Endosomal sorting of MOR, based on a unique leucine-based sequence, can dynamically regulate its responses to different agonists at cellular and organismal levels (Williams et al., 2013; Bowman et al., 2015; Weinberg et al., 2017) MOR undergoes rapid phosphorylation and dephosphorylation in response to agonists, and heterologous manipulation of this phosphorylation regulates its plasma membrane localization, trafficking, and signaling (Feng et al., 2011; Just et al., 2012; Bowman et al., 2015; Halls et al., 2016; Arttamangkul et al., 2018).

In this study, we used a real-time live-cell-imaging assay that resolves MOR recycling at single-event resolution to study the homologous signals that allow self-regulation of endosomal sorting of MOR. We show that activation of MOR initiates downstream signaling cascades that enhance postendocytic receptor recycling back to the cell surface. The signaling cascade requires the $\mathrm{G}_{\beta \gamma}$-activated phospholipase $\mathrm{C}$ (PLC)/protein kinase $\mathrm{C}$ (PKC) signaling pathway, which results in the phosphorylation of serine 363 on MOR's C-terminal tail. This phosphorylation is required for increased MOR recycling. The positive feedback loop that regulates MOR endocytic trafficking that we describe here may serve as a template for similar adaptive self-regulatory mechanisms for many GPCRs.

\section{Materials and Methods}

Reagents, Constructs, and Cells. [D-Ala2, N-MePhe4, Gly-ol]enkephalin (DAMGO; used at $10 \mu \mathrm{M})$, naltrexone (10 $\mu \mathrm{M})$, pertussis toxin $(100 \mathrm{ng} / \mathrm{ml}$, overnight treatment), forskolin $(10 \mu \mathrm{M})$, KT5720 (1 $\mu \mathrm{M}$ ), myr-SIRKALNILGYPDYD-OH (mSIRK; $10 \mu \mathrm{M}$ ), U73122 $(10 \mu \mathrm{M})$, and chelerythrine $(5 \mu \mathrm{M})$ were purchased from SigmaAldrich (St. Louis, MO). Gallein $(20 \mu \mathrm{M}, 30$ minute treatment), U73343 $(10 \mu \mathrm{M})$, and Gö6983 $(5 \mu \mathrm{M})$ were purchased from Tocris Bioscience. Compound $12155(10 \mu \mathrm{M})$ was provided by Dr. Alan Smrcka (University of Michigan). Stable nonclonal HEK293 cells (American Type Culture Collection CRL-1573) expressing superecliptic phluorin ( $\mathrm{SpH})-\mathrm{MOR}$ were selected in Geneticin (Invitrogen) and grown in Dulbecco's modified Eagle's medium (Hyclone) $+10 \%$ fetal bovine serum (Gibco). The SpH-MOR-S363A point mutant thereof has been described previously (Soohoo and Puthenveedu, 2013; Bowman et al., 2015). SpH-MOR-S363D was generated by Q5 Site-Directed Mutagenesis Kit (New England Biolabs) using ACAGCAAAACGA TGCTCGAATCCG as the forward primer and TCGATTGTGGAGGAA GTTG as the reverse primer.

Live Cell Imaging. Cells were passed to $25-\mathrm{mm}$ glass coverslips coated with poly-D-lysine and imaged 2 days later. Cells were imaged live in Leibovitz L15 imaging medium (Gibco) and $1 \%$ fetal bovine serum at $37^{\circ} \mathrm{C}$ in a temperature- and $\mathrm{CO}_{2}$-controlled chamber. $\mathrm{A}$ Nikon Eclipse Ti automated inverted microscope with a $60 \times / 1.49$ N.A. total internal reflection fluorescence objective and confocal $20 \times / 0.75$ N.A. objective was used for imaging. Images were acquired with an iXon+ 897 electron-multiplying charge-coupled device camera (Andor, Belfast, UK) with a solid-state laser of $488 \mathrm{~nm}$ as a light source.
A

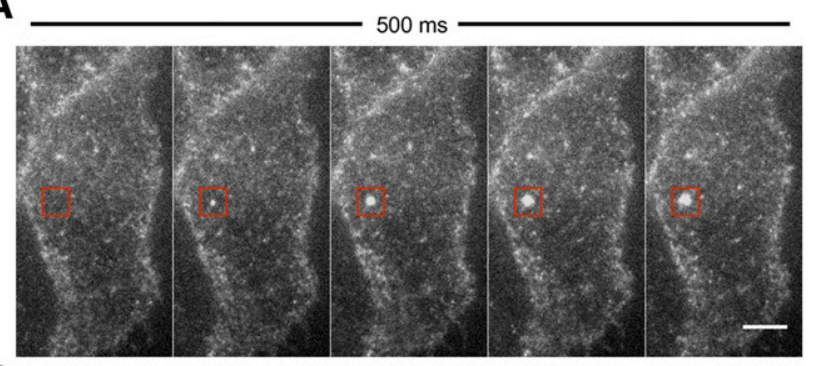

B

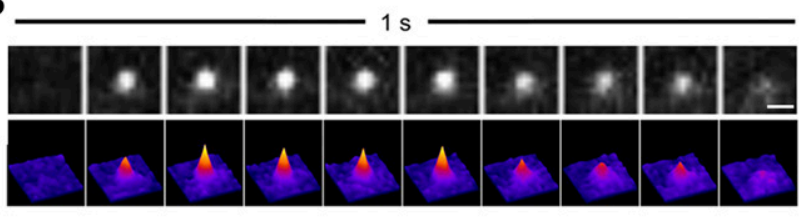

C

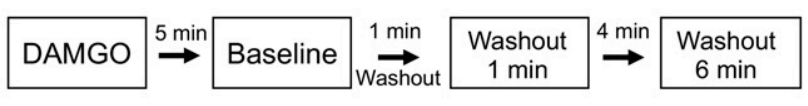

D

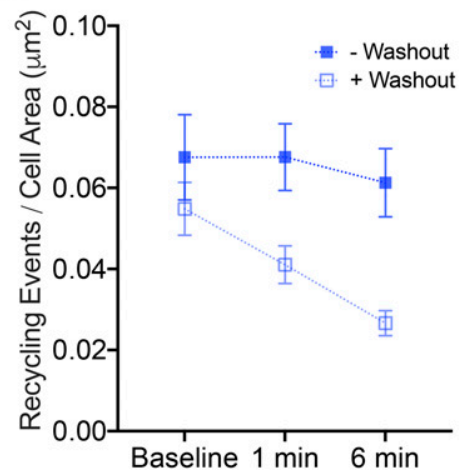

E

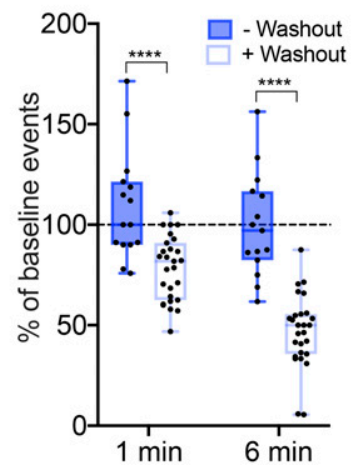

Fig. 1. The opioid agonist DAMGO increases postendocytic recycling of MOR. (A) HEK293 cell expressing SpH-MOR imaged with total internal reflection fluorescence microscopy after DAMGO addition. The appearance of an individual exocytic recycling event is denoted by red boxes. Images are $100 \mathrm{~ms}$ apart. Scale bar, $5 \mu \mathrm{m}$. (B) Profile of an individual exocytic event (puff) over $1 \mathrm{~s}$. Frames are $100 \mathrm{~ms}$ apart. Scale bar, $1 \mu \mathrm{m}$. The event begins as a defined spot of fluorescence intensity that appears suddenly. The fluorescence diffuses on the cell membrane as shown by the heat map surface plot. (C) Experimental paradigm to study MOR postendocytic recycling. (D) The number of recycling events normalized to cell area (square micrometer) over time \pm DAMGO washout after the baseline recording. In the -washout condition, $P>0.999$ for baseline versus +1 minute and $P=0.306$ for baseline versus +6 minutes $(n=15$ cells $)$. In the +washout condition, $* * * * P<0.0001$ for both baseline versus 1-minute washout and baseline versus 6 -minute washout ( $n=27$ cells). Mean and S.E.M. are plotted for each time point. (E) The percentage of recycling events in each condition ( \pm washout) was normalized to the baseline events for each condition. ${ }^{* * * *} P<0.0001$ for - washout 1 minute versus +washout 1 minute. ${ }^{* * * * *} P<0.0001$ for -washout 6 minutes versus +washout 6 minutes ( - washout: $n=15$ cells; + washout: $n=27$ cells.) Box and whisker plots are shown with all points from each condition.

Images were scrambled using a scrambler.py script (https://gist. github.com/SavinaRoja/1629319) before analyzing them in FIJI (National Institutes of Health) (Schindelin et al., 2012).

Quantification of Individual Recycling Events. HEK293 cells stably expressing SpH-MOR or its mutants were treated with DAMGO for 5 minutes to induce receptor clustering and internalization at $37^{\circ} \mathrm{C}$. Receptor clustering was visualized by acquiring an image every 3 seconds for 5 minutes. A baseline recycling movie was acquired at $10 \mathrm{~Hz}$ for 1 minute using total internal reflection fluorescence microscopy, followed by a washout with antagonist (naltrexone). 
A

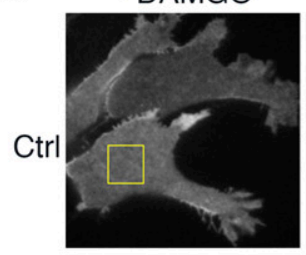

+ DAMGO
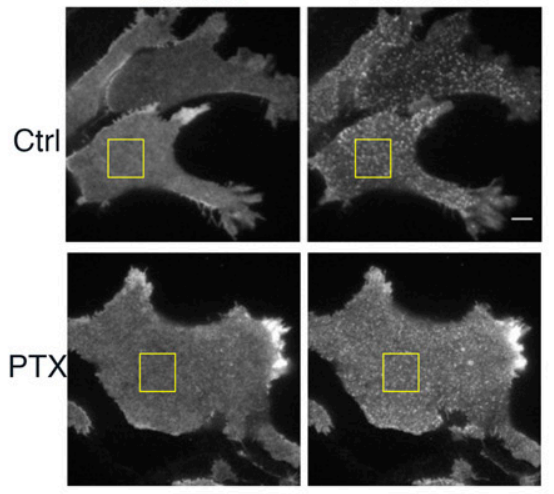

D

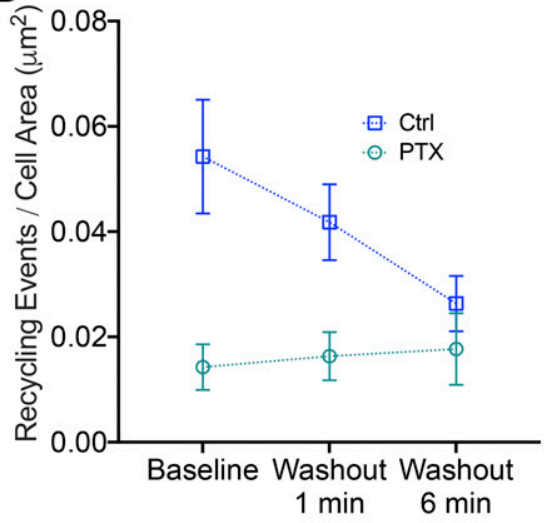

B
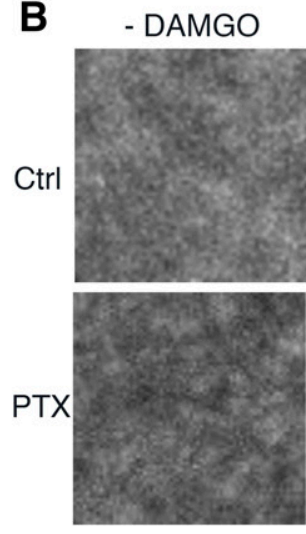

E

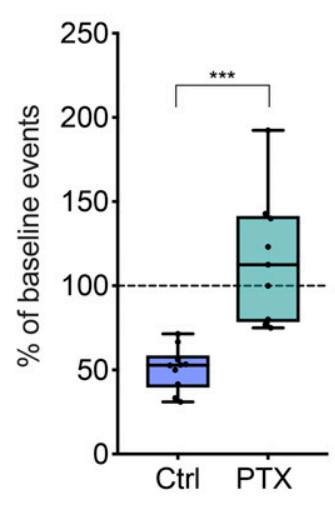

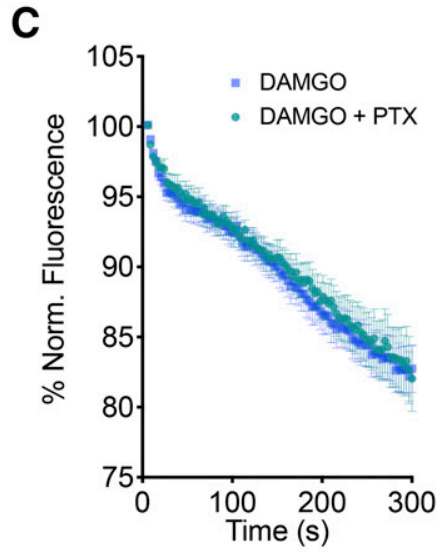

$\mathbf{F}$

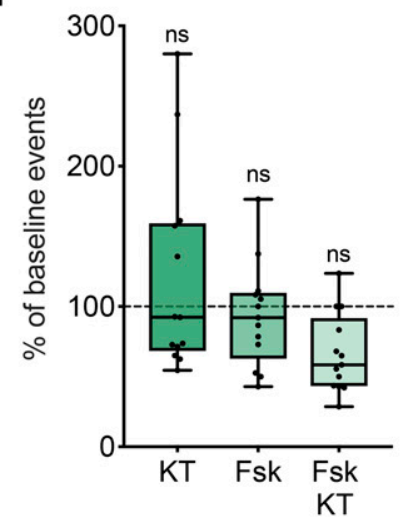

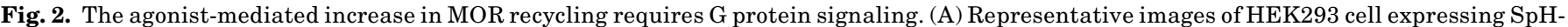

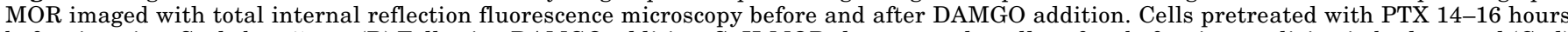

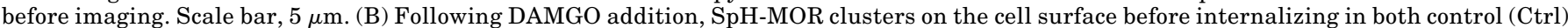

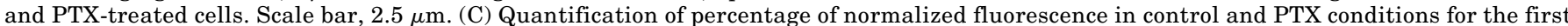

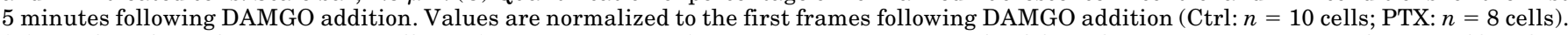

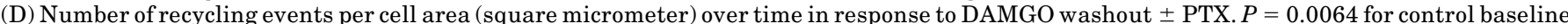

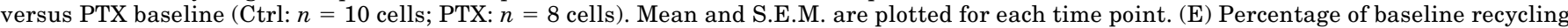

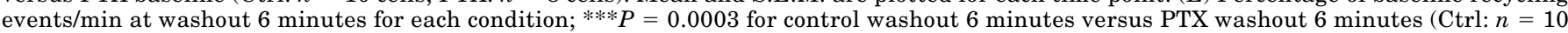

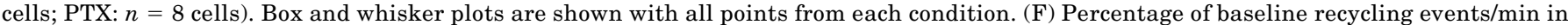

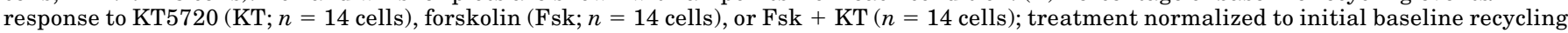

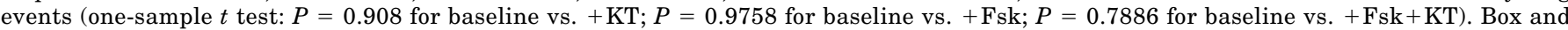
whisker plots are shown with all points from each condition. ns, not significant.

Subsequent movies were collected 1 and 6 minutes after washout. The number of individual exocytic recycling events in each movie was manually scored. The box plots display the median and the entire range with outliers excluded. To evaluate the effect of agonist washout on the frequency of recycling events between treatment conditions, two-way repeated-measures ANOVAs were conducted on the raw recycling rates followed by post hoc comparisons of means within the treatment condition to the baseline recycling rate. These tests were conducted separately for each of the sets of conditions shown in Figs. 1D; 2D; 3, A and C; and 4C. Supplemental Figure 2. To compare the magnitude of the washout effect between treatment conditions, recycling events for each cell were normalized to that cell's recycling rate in the baseline movie. The mean normalized recycling rate was then compared between conditions of interest using either a one-way ANOVA followed by the indicated post hoc comparisons using Dunnett's multiple comparisons correction (Figs. 1E, 3D, and 4D) or a single paired Student's $t$ test when only two conditions were evaluated (Figs. 2E and 3B). When testing whether a pharmacological treatment was able to perturb the basal rate of recycling, the normalized recycling events were compared with a theoretical mean of $100 \%$ using a single-sample Student's $t$ test (Figs. $2 \mathrm{~F}$ and 3, $\mathrm{A}$ and $\mathrm{B})$.
Ensemble Recycling Assay. To measure ensemble recycling, receptor surface levels were imaged using confocal microscopy with a $20 \times$ objective and 488 -nm laser. Images were collected at 30 -second intervals for 20 different fields. Baseline recordings for 2 minutes (four frames) were collected before the addition of DAMGO. After the addition of agonist, images were collected for 15 minutes. After 15 minutes, agonist was removed. The cells were rinsed with fresh imaging medium, and antagonist (naltrexone) was added to the new medium. After agonist washout, images were collected for another 15 minutes. Fluorescence intensities were corrected by a background threshold and normalized by the average fluorescence of the baseline four frames collected before DAMGO treatment. Surface fluorescence analysis was conducted using an ImageJ Macro automated script (National Institutes of Health) (https://zenodo.org/record/2645754). Fluorescence recovery/loss ratios after washout were quantified by normalizing the fluorescence values after washout to the total fluorescence lost before washout. Cell fields that did not respond to DAMGO treatment were excluded from analysis. Statistical significance was determined by using a one-way ANOVA comparing endpoints of all conditions to the control condition, followed by post hoc comparisons between all means (Fig. 3H). 
A

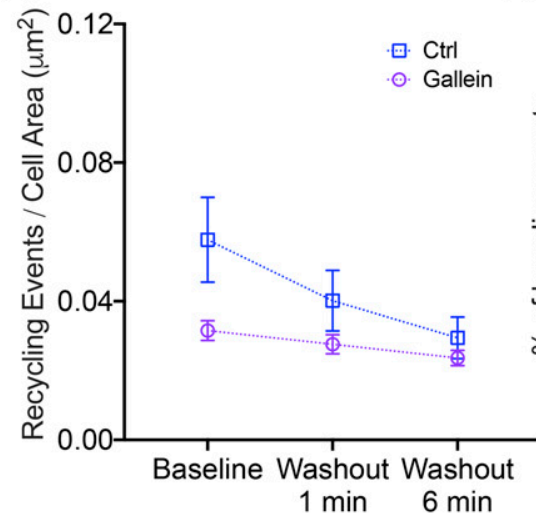

B

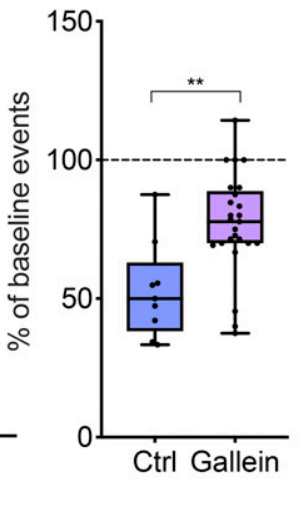

C

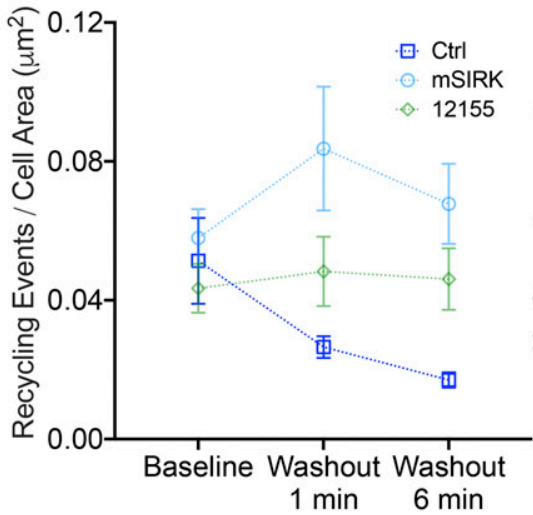

D

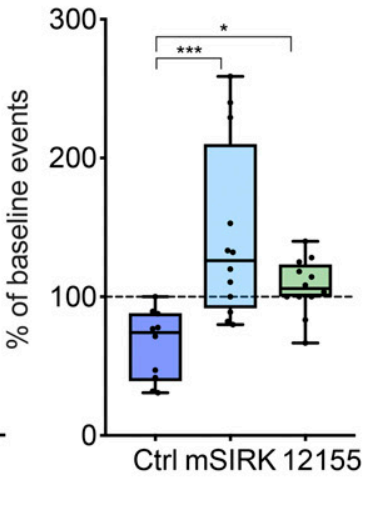

E
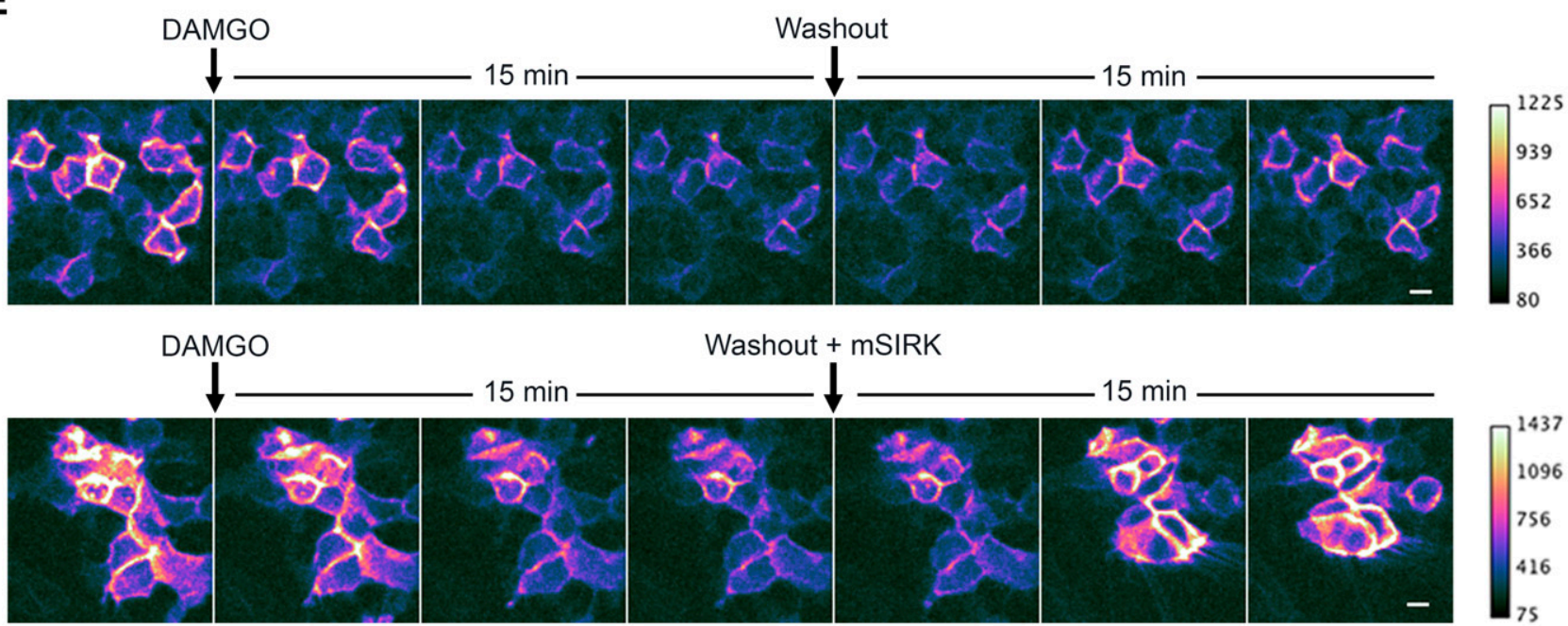

F

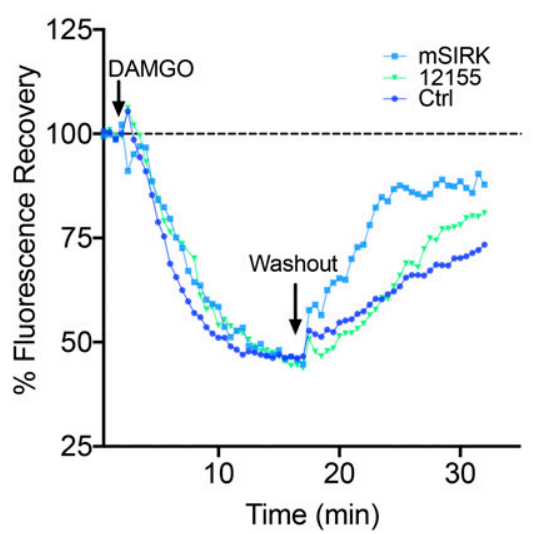

G

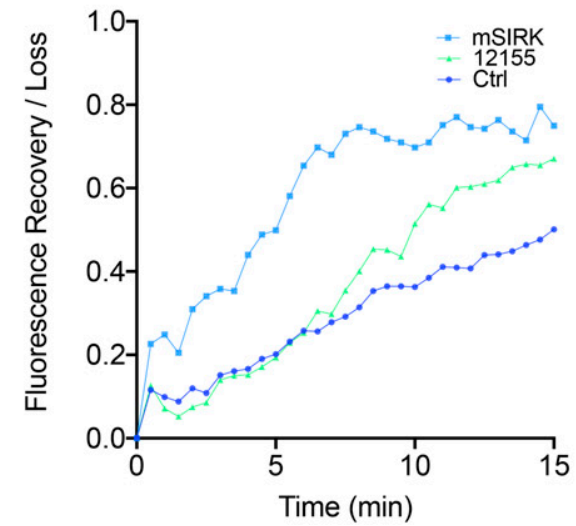

H

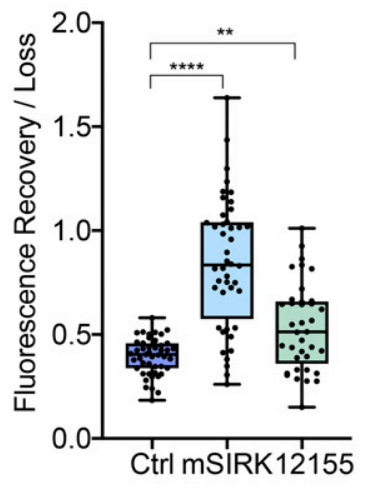

Fig. 3. $\mathrm{G}_{\beta \gamma}$ activation is required and sufficient to increase MOR recycling. (A) Number of MOR recycling events per cell area (square micrometer) over time in response to a DAMGO washout in control $(\mathrm{Ctrl})$ and gallein conditions. Cells were treated with gallein 30 minutes prior to imaging. $P=0.0046$ for control baseline versus gallein baseline (Ctrl: $n=9$ cells; gallein: $n=25$ cells). Mean and S.E.M. are plotted for each time point. (B) Percentage of baseline recycling events/min at washout 6 minutes for each condition: $P=0.0015$ for control washout 6 minutes versus gallein washout 6 minutes $($ Ctrl: $n=9$ cells; gallein: $n=25$ cells). Box and whisker plots are shown with all points from each condition. (C) Number of MOR recycling events per cell area (square micrometer) over time in response to a DAMGO washout in control, mSIRK, and 12155 conditions. Cells were treated acutely with mSIRK or 12155 during the washout. Mean and S.E.M. are plotted for each time point (Ctrl: $n=10$ cells; mSIRK: $n=12$ cells; $12155: n=12$ cells). (D) Percentage of baseline recycling events/min at washout 1 minute for each condition: $* * * P=0.0003$ for control washout 1 minute versus mSIRK washout 1 minute; ${ }^{*} P=$ 0.0488 for control washout 1 minute versus 12155 washout 1 minute (Ctrl: $n=10$ cells; mSIRK: $n=12$ cells; $12155: n=12$ cells). Box and whisker plots are shown with all points from each condition. (E) Changes in surface MOR fluorescence over time measured after DAMGO addition and washout. MOR fluorescence decreased upon receptor internalization after DAMGO addition and returned upon recycling after DAMGO washout. $\mathrm{G}_{\beta \gamma}$ activation by either mSIRK or 12155 increased the rate of recovery of fluorescence. Scale bar, $10 \mu \mathrm{m}$. (F) Quantification of fluorescence recovery over 30 minutes following DAMGO treatment normalized to the baseline fluorescence. (G) Quantification of fluorescence recovery normalized to the fluorescence loss before washout for control, mSIRK, or 12155 conditions. (H) Box and whisker plots showing the fluorescence after 15 minutes of agonist washout in control, mSIRK, or 12155 conditions. $G_{\beta \gamma}$ activation by either mSIRK or 12155 increased the receptors recycled; $* * * * P<0.0001$ for control versus mSIRK, $* * P=0.0058$ for control versus 12155 (Ctrl: $n=48$ fields; mSIRK: $n=44$ fields; $12155: n=38$ fields, all across three independent experiments). 


\section{Results}

The Opioid Agonist DAMGO Increases Postendocytic Recycling of MOR. To determine if the signals downstream of activated MOR regulate its own postendocytic recycling, we quantitated MOR recycling using a live cell imaging assay that resolves individual MOR recycling events without the confounding effects of endocytosis. To visualize recycling events, MOR was $\mathrm{N}$-terminally tagged with a $\mathrm{pH}$-sensitive GFP (SpH) (Sankaranarayanan et al., 2000). HEK293 cells stably expressing MOR that was N-terminally tagged with the $\mathrm{pH}$-sensitive GFP superecliptic phluorin ( $\mathrm{SpH}-\mathrm{MOR}$ ) were imaged using total internal reflection fluorescence microscopy. SpH-MOR on the cell surface is fluorescent and readily detectable. After activation, receptors are internalized into acidic endosomal compartments where the $\mathrm{SpH}$ fluorescence is quenched. When recycling vesicles containing receptors fuse back to the plasma membrane during an exocytic event, the $\mathrm{SpH}$ fluorescence is exposed to the neutral $\mathrm{pH}$ of the extracellular medium and is therefore dequenched. This coordinated dequenching generates a characteristic "puff" of fluorescence that is readily detectable (Supplemental Movie 1). We and others have extensively characterized these and similar puffs previously and confirmed that they represent individual vesicle fusion events during receptor recycling (e.g., Yudowski et al., 2006; Bowman et al., 2015; Logan et al., 2017).

Individual fusion events were readily observed after cells expressing SpH-MOR were treated with the opioid agonist DAMGO for 5 minutes (Fig. 1, A and B). Agonist-containing medium was washed out and replaced with medium containing naltrexone. Recycling movies were recorded 1 and 6 minutes after this washout (Fig. 1C) and the number of individual events was normalized to the area in each cell. The raw recycling rate per cell area \pm washout was determined for each recycling movie (Fig. 1D). This approach allowed us to follow changes in the same cells over time and to normalize the raw values to the baseline recycling rate in each condition for each cell (Fig. 1E). When compared with cells continuously exposed to agonist for similar times (Fig. 1D), the number of MOR recycling events decreases from baseline following agonist removal $(P<0.0001)$. Additionally, the normalized data matched at the same time points indicate statistically significant differences in recycling at 1 minute $(P<0.0001)$ and 6 minutes $(P<0.0001)$ for - washout versus + washout (Fig. 1E). These results indicate that MOR signaling positively regulates MOR recycling.

The Agonist-Mediated Increase in MOR Recycling Rate Requires G Protein Signaling. To address which signals downstream of receptor activation are involved in regulating postendocytic MOR recycling, we first probed the role of $\mathrm{G}$ proteins that couple to MOR by using the G protein inhibitor pertussis toxin (PTX). In SpH-MOR cells treated with PTX 14-16 hours before imaging, DAMGO-induced MOR clustering into endocytic domains was not inhibited (Fig. 2, A and B), consistent with previous studies (Halls et al., 2016; (Gondin et al., 2019)). Fluorescence values normalized to the first frames after DAMGO addition were quantified for control and PTX-treated cells as an index of receptor internalization. The decrease in fluorescence over the first 5 minutes after DAMGO addition was not different between control and PTXtreated cells, suggesting that endocytosis of the receptor was not altered due to PTX treatment (Fig. 2C). When individual recycling events were quantified in the PTX condition, however, the baseline recycling rate was decreased compared with control $(P=0.0064)$, as shown in the summary data (Fig. 2D). The full box plots and statistical comparisons are shown in the corresponding panel of Supplemental Fig. 2 (Supplemental Fig. 2B). Following agonist washout, the recycling rate was unchanged compared with before washout in the PTX condition (Fig. 2D). The normalized recycling for each baseline condition indicates a statistically significant change in the percentage of initial recycling between \pm PTX at the time point 6 minutes after washout $(P=0.0003)$. These results show that agonist-mediated increase in MOR recycling requires $\mathrm{G}$ protein signaling.

Since $G_{\alpha \mathrm{i}}$ negatively regulates protein kinase A (PKA) through its primary effector adenylate cyclase, we next tested whether PKA activity changed MOR recycling. PKA inhibition by KT5720, an acute PKA inhibitor, did not change MOR recycling compared with untreated cells (Fig. 2F). Further, neither forskolin, which activates adenylate cyclase, thereby increasing cAMP and activating PKA, nor subsequent inhibition of PKA in the same cells changed MOR recycling rates (Fig. 2F). These results suggest that, in our cells, cAMP and PKA signaling downstream of $\mathrm{G}_{\alpha \mathrm{i}}$ does not regulate MOR recycling.

$\mathbf{G}_{\beta \gamma}$ Signaling Is Required and Sufficient to Increase Postendocytic Recycling of MOR. We next tested whether $\mathrm{G}_{\beta \gamma}$ activation regulates MOR recycling. To test whether $\mathrm{G}_{\beta \gamma}$ activation was required, we inhibited $\mathrm{G}_{\beta \gamma}$ using gallein, a small-molecule inhibitor of $\mathrm{G}_{\beta \gamma}$ activation, and measured MOR recycling (Bonacci et al., 2006). In cells incubated with gallein 30 minutes prior to imaging, receptor-mediated clustering and endocytosis were not altered with gallein treatment (Supplemental Fig. 1A), but baseline recycling decreased compared with that of untreated cells $(P=$ 0.0046) (Fig. 3A). Agonist washout in gallein-treated cells did not decrease recycling to the same extent compared with the control (Fig. 3B), suggesting that the agonist-mediated increase in MOR recycling requires $\mathrm{G}_{\beta \gamma}$ signaling. To test whether $\mathrm{G}_{\beta \gamma}$ signaling was sufficient to increase MOR recycling, we activated $\mathrm{G}_{\beta \gamma}$ in the absence of agonist and measured MOR recycling. We used mSIRK, a cell-permeable peptide activator of $\mathrm{G}_{\beta \gamma}$, and the compound 12155, a cell-permeable small-molecule activator of $\mathrm{G}_{\beta \gamma}$ (Goubaeva et al., 2003; Lehmann et al., 2008; Surve et al., 2014), to activate $\mathrm{G}_{\beta \gamma}$. Both $\mathrm{G}_{\beta \gamma}$ activators increased the rate of MOR recycling at 1 and 6 minutes after washing out DAMGO compared with control cells (Fig. 3, C and D). Interestingly, 12155 increased MOR recycling to a lesser degree than mSIRK, which may reflect different $\mathrm{G}_{\beta \gamma}$ activation mechanisms by these two drugs (Bonacci et al., 2006; Surve et al., 2014). Together, these results indicate that $\mathrm{G}_{\beta \gamma}$ signaling is required and sufficient to enhance MOR recycling back to the cell surface.

Our results indicating that $\mathrm{G}_{\beta \gamma}$ signaling is required and sufficient to enhance MOR recycling were also confirmed by measuring surface levels of SpH-MOR at an ensemble level in live cells over time. Since $\mathrm{SpH}$ is only fluorescent on the cell surface and not when the receptor is in endosomes, the fluorescence signal accurately measures surface levels of MOR (Yudowski et al., 2009; Vistein and Puthenveedu, 2013). After DAMGO addition, there is a significant decrease in fluorescence intensity $(\sim 50 \%)$ as predicted due to receptor internalization. This level reaches a plateau around 
10 minutes, which indicates a steady state between endocytosis and recycling. When agonist is removed in a washout, the steady state is shifted by removing the contribution of endocytosis. This results in an increase in fluorescence intensity, which allows us to capture the contribution of receptor recycling.

Multiple fields $(n>15)$ of cells expressing SpH-MOR were selected for each experiment. After collecting a 2-minute baseline signal for each field, DAMGO was added, and the fluorescence signal was recorded for 15 minutes until the signal plateaued. After 15 minutes, the medium containing DAMGO was washed out and replaced with fresh medium containing antagonist (naltrexone) and $\pm \mathrm{mSIRK}$ or \pm 12155 . The recovery of fluorescence signal was measured for 15 minutes after washout to determine a measurement of ensemble MOR recycling across cells (Fig. 3E). In the $\mathrm{G}_{\beta \gamma^{-}}$ activating conditions, we observed an initial spike in fluorescence signal consistent with the increase we observed in the orthogonal puffs assay (Fig. 3, F and G). Overall the increase in fluorescence signal indicates an increase in the number of receptors recycled back to the cell surface in response to sustained $\mathrm{G}_{\beta \gamma}$ activation (Fig. $3 \mathrm{H}$ ). These results strongly support that $\mathrm{G}_{\beta \gamma}$ signaling is required and sufficient to increase MOR recycling.

MOR Regulates Its Own Recycling via Phospholipase $C$, Protein Kinase $C$, and Serine 363. To determine the signals downstream of $\mathrm{G}_{\beta \gamma}$ activation that regulated MOR recycling, we first examined the role of $\mathrm{PLC}$, which is activated by $\mathrm{G}_{\beta \gamma}$. To test whether PLC inhibition changed DAMGOmediated regulation of MOR recycling, we acutely inhibited PLC with U73122 and measured the change in MOR recycling from baseline recycling before PLC inhibition. U73122 decreased MOR recycling even in the presence of DAMGO, whereas U73343, an inactive control, did not change MOR recycling compared with baseline (Fig. 4A). These results suggest that PLC activation is required for the DAMGOmediated increase in MOR recycling. We next tested whether $\mathrm{PKC}$ signaling was required to increase MOR recycling in the presence of DAMGO. To do this, we acutely inhibited PKC by treating cells with chelerythrine or Gö6983 and measured the change in MOR recycling from baseline recycling before PKC inhibition (Fig. 4B). MOR recycling was reduced when PKC was inhibited, suggesting that PKC increased MOR recycling.

Because the C-terminal tail of MOR contains predicted PKC phosphorylation sites (Doll et al., 2011), we tested whether the receptor itself was a target of DAMGO-mediated regulation of MOR recycling. Specifically, we focused on serine 363, a putative $\mathrm{PKC}$ phosphorylation site, as a potential regulator of $\mathrm{PKC}$ signaling. To test whether serine 363 was required for DAMGO-mediated homologous regulation of MOR recycling, we mutated serine 363 on MOR to alanine. Receptor-mediated clustering and endocytosis were not different between S363A and wild-type (WT) MOR (Supplemental Fig. 1B). In cells expressing this mutant construct (S363A), however, the number of recycling events was decreased compared with the cells expressing the wild-type MOR (Fig. 4C). The reduction in recycling seen upon washout of drug was smaller for S363A compared with the wild-type MOR but was not fully abolished, suggesting that other residues or mechanisms may be involved in the regulation of DAMGO-mediated recycling (Fig. 4D). Further, the recycling of S363A was also insensitive to $\mathrm{G}_{\beta \gamma}$ inhibition by gallein (Fig. $4, \mathrm{C}$ and D). Together, these data indicate that phosphorylation at serine 363 is required to increase MOR recycling after activation.

To test if phosphorylation at serine 363 was sufficient to increase MOR recycling in the absence of MOR activation, we examined recycling in cells expressing a phosphomimetic MOR mutant where serine 363 was mutated to an aspartate (S363D). Baseline recycling was not different between the WT MOR and the S363D mutant. However, unlike WT MOR, recycling of the $\mathrm{S} 363 \mathrm{D}$ mutant did not decrease from baseline after agonist washout (Fig. 4C), suggesting that the phosphomimetic mutation was sufficient to keep MOR recycling at a high level even after agonist washout. Together, our results suggest a model of homologous regulation of MOR recycling, where MOR activation phosphorylates the receptor at serine 363 via $\mathrm{G}_{\beta \gamma}$ signaling, PLC, and PKC and increases MOR recycling back to the cell surface (Fig. $4 \mathrm{E}$ ).

\section{Discussion}

We identify a positive feedback mechanism that mediates homologous regulation of MOR recycling. Activation of MOR initiates a signaling cascade via $\mathrm{G}_{\beta \gamma}$ and $\mathrm{PKC}$ that increases the rate of MOR recycling. This increase in recycling requires the phosphorylation of MOR at serine 363, a site that can be directly phosphorylated by PKC.

Our results provide new information on how receptor phosphorylation can regulate MOR trafficking. Phosphorylation of MOR, mainly at the TSST (residues 354-357) and the STANT (residues 375-379) motifs, by multiple kinases, has been studied extensively for its role in receptor internalization and desensitization (Williams et al., 2013; Arttamangkul et al., 2018; Miess et al., 2018; Kliewer et al., 2019). PKC can phosphorylate MOR at multiple sites, but the residues that are phosphorylated in vivo and the role of PKC phosphorylation in regulating MOR trafficking and function are still being investigated (Doll et al., 2011; Feng et al., 2011; Yousuf et al., 2015). The serine 363 residue that we identified as critical for regulating MOR recycling can be phosphorylated in vitro by $\mathrm{PKC} \varepsilon$, although whether this is the primary enzyme that phosphorylates it in vivo is not clear (Doll et al., 2011; Feng et al., 2011). PKC activation by MOR on the plasma membrane varies between different agonists used (Halls et al., 2016). Further, activation of PKC downstream of $\mathrm{G}_{\beta \gamma}$ can regulate receptor localization to different domains on the plasma membrane (Halls et al., 2016). Once internalized, the phosphorylation of MOR at specific residues can determine receptor sorting between Rab4- and Rab11-dependent recycling compartments (Wang et al., 2008). In this context, the rate and extent of dynamic changes in phosphorylation at S363 of MOR is not clear. In biochemical assays, the bulk levels of S363 phosphorylation do not change after agonist treatment (Lau et al., 2011; Moulédous et al., 2015). Considering our data, that this site is required and sufficient to mediate agonist-mediated increase in MOR recycling, it is possible that phosphorylation at this site is locally and transiently regulated in response to receptor activation.

Regulation of MOR recycling by $\mathrm{G}_{\beta \gamma^{-}}$, PLC-, and PKCmediated receptor phosphorylation is a novel example of homologous regulation of GPCR recycling. Receptor phosphorylation has been studied mainly in the context of receptor desensitization and endocytosis, but phosphorylation can regulate the recycling of other receptors such as the $\beta$ 
A

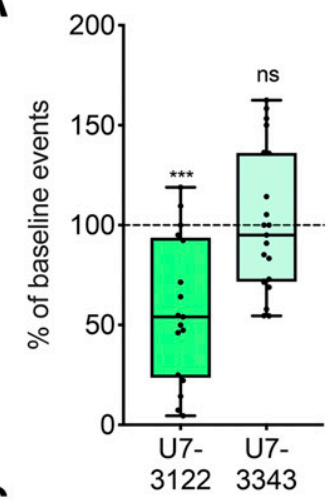

B

C

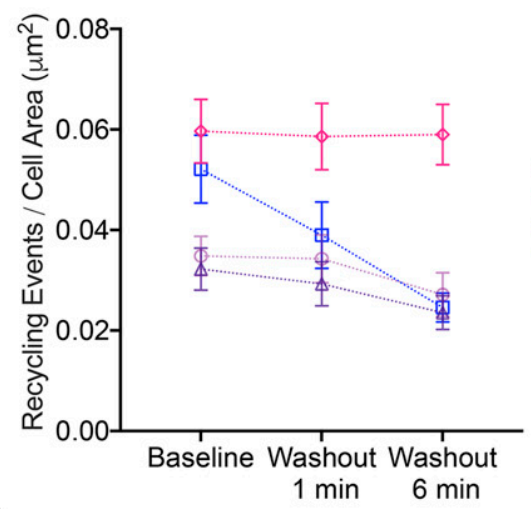

D

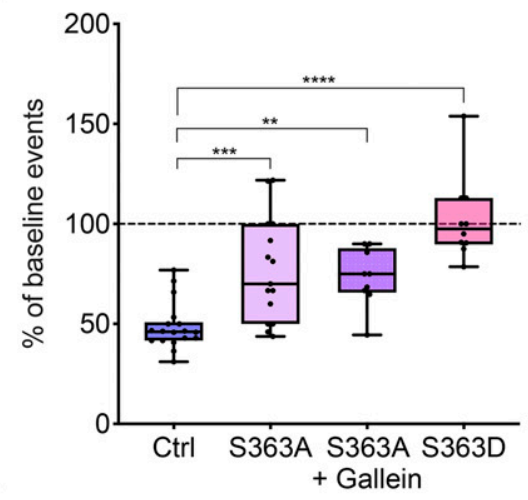

E

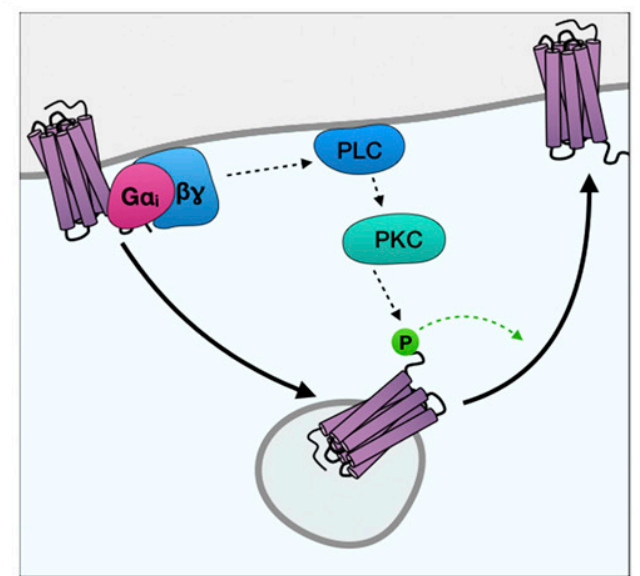

Fig. 4. Homologous regulation of MOR recycling by MOR phosphorylation at serine 363. (A) Percentage of baseline recycling events/min in response to U73122 ( $n=17$ cells $)$ or U73343 $(n=21$ cells $)$ treatment normalized to initial baseline recycling events. One-sample $t$ test: $* * * P=$ 0.0002 for baseline versus U73122; $P=0.8910$ for baseline versus U73343. Box and whisker plots are shown with all points from each condition. adrenergic receptors. In the case of the $\beta 2$-adrenoreceptor $(\beta 2 \mathrm{AR})$, a prototypical $\mathrm{G}_{\alpha \mathrm{s}}$-coupled receptor, receptor recycling is regulated by receptor phosphorylation downstream of receptor signaling. However, unlike for MOR, agonist stimulation decreased the postendocytic recycling of $\beta 2 \mathrm{AR}$. This decrease required PKA-mediated phosphorylation of $\beta 2 \mathrm{AR}$ on serine 345/346 (Yudowski et al., 2009; Vistein and Puthenveedu, 2013). PKA signaling, however, does not play a role in MOR recycling in our cells (Fig. $2 \mathrm{~F}$ ). MOR recycling in striatal neurons has been reported to be inhibited by forskolin, although PKA was not directly tested (Roman-Vendrell et al., 2012). This difference could reflect differences in mechanisms of recycling of $\beta 2 \mathrm{AR}$ and $\mathrm{MOR}$, which might also differ between cell types. In HEK cells, $\beta 2 A R$ recycles via a specialized set of endosomal tubules, termed ASRT tubules, characterized by the presence of an actin-SNX-retromer complex (Puthenveedu et al., 2010; Temkin et al., 2011). $\beta 2 \mathrm{AR}$ sorting into these tubules requires the interaction of a C-terminal post-synaptic density-95/disc large tumor suppressor/zonula occludens-1 (PDZ) ligand sequence with a set of PDZcontaining proteins, which ultimately link the receptor to the endosomal actin cytoskeleton (Temkin et al., 2011). MOR seems to recycle via a distinct mechanism, although the mechanism itself is not understood. MOR recycling requires a unique "bileucine" sequence on the receptor C-terminal tail. There is no evidence that this sequence interacts with the PDZ-containing proteins or actin. Further, $\beta 2 \mathrm{AR}$ and MOR might also use different sets of Rab proteins to recycle (Wang et al., 2008). The use of both $\mathrm{G}_{\alpha}$ - and $\mathrm{G}_{\beta \gamma}$-mediated pathways to phosphorylate receptor cargo via distinct kinases to regulate recycling of receptors through potentially distinct pathways suggests that homologous regulation of receptor recycling by modifying receptor phosphorylation states is a conserved mechanism on a global level, although the specific mechanisms might vary between different GPCRs.

The changes we observe in MOR recycling based on PKCmediated receptor phosphorylation could have direct effects on MOR signaling and function. Many canonical GPCRs have been shown to signal from endosomes in the recent past, raising the idea that endosomal signaling is the norm rather than the exception (Calebiro et al., 2009; Vilardaga et al., 2014; Bowman et al., 2016; Jensen et al., 2017; Eichel and von Zastrow, 2018). When localized to specific microdomains on

(B) Percentage of baseline recycling events/min in response to chelerythrine $(n=20$ cells $)$ or Gö6983 ( $n=21$ cells $)$ treatment normalized to initial baseline recycling events. One-sample $t$ test: $* * * P=0.0007$ for baseline versus chelerythrine; **** $P<0.0001$ for baseline versus Gö6983. Box and whisker plots are shown with all points from each condition. (C) Number of recycling events per cell area (square micrometer) over time in response to DAMGO washout in control (Ctrl), S363A, S363A + gallein, or S363D conditions. $P=0.0392$ for control baseline vs. S363A baseline; $P=0.0429$ for control baseline vs. S363A + gallein baseline; $P=0.7203$ for control baseline vs. S363D baseline; $P=0.9391$ for S363D baseline vs. S363D washout 6 minutes (Ctrl: $n=18$ cells; S363A: $n=15$ cells; S363A + gallein: $n=19$ cells; S363D: $n=10$ cells). Mean and S.E.M. are plotted for each time point. (D) Percentage of baseline recycling events/min at washout 6 minute for each condition: $* * * P=0.0003$ for control washout 6 minute versus $\mathrm{S} 363 \mathrm{~A}$ washout 6 minutes; $* * P=0.0077$ for control washout 6 minutes versus S363A + gallein washout 6 minutes; $* * * * P<0.0001$ for control washout 6 minutes versus S363D washout 6 minutes (Ctrl: $n=18$ cells; S363A: $n=15$ cells; S363A + gallein: $n=19$ cells; S363D: $n=10$ cells). Box and whisker plots are shown with all points from each condition. (E) Proposed model of self-regulation of postendocytic recycling of MOR. ns, not significant. 
the endosome, $\beta 2 \mathrm{AR}$ activates the transcription of a complement of genes that are distinct from those activated by signaling from the plasma membrane (Tsvetanova and von Zastrow, 2014; Bowman et al., 2016). Endosomal signaling of the neurokinin-1 receptor in spinal neurons, or the calcitonin gene-related peptide-targeted calcitonin-like receptor, contributes to nociception (Jensen et al., 2017; Yarwood et al., 2017; Weinberg et al., 2019). Whether MOR signaling from endosomes has a distinct signaling consequence is less clear. However, increased recycling of MOR, induced by neurokinin1 receptor signaling, can decrease acute tolerance to opioids, suggesting that the rate of recycling can regulate opioid physiology (Bowman et al., 2015). PKC-mediated phosphorylation of MOR could therefore serve as a convergence point for both homologous regulation of MOR recycling by $\mathrm{G}_{\beta \gamma}$ and heterologous regulation by other signaling pathways. Importantly, considering emerging data that the precise location of receptors could dictate PKC-mediated phosphorylation of MOR, this could regulate both the rate of resensitization as well as the spatial encoding of opioid signaling, which has emerged as an exciting area of study in the recent past (Sorkin and von Zastrow, 2009; Tsvetanova and von Zastrow, 2014; Bowman et al., 2016; Eichel and von Zastrow, 2018). Similar feedback loops may serve as templates for adaptive selfregulation of signaling for many GPCRs, although the specific mechanisms may vary between different receptors. Understanding the regulation of GPCR trafficking, using assays that can directly detect and measure these dynamic events in real time, will allow us to better analyze the relationship between receptor trafficking and the spatiotemporal aspects of GPCR signaling.

\section{Acknowledgments}

The authors thank Drs. Shanna Bowman, Amynah Pradhan, Stefan Schulz, Peter Friedman, Alan Smrcka, and Mark von Zastrow for reagents, comments, and suggestions.

\section{Authorship Contributions}

Participated in research design: Kunselman, Zajac, Weinberg, Puthenveedu.

Conducted experiments: Kunselman, Zajac, Weinberg,

Puthenveedu.

Performed data analysis: Kunselman, Zajac, Weinberg,

Puthenveedu.

Wrote or contributed to the writing of the manuscript: Kunselman, Zajac, Weinberg, Puthenveedu.

Note Added in Proof-A typo was found in the (NIH) National Institute of General Medical Sciences grant number in the Fast Forward version published October 1, 2019. The funding footnote has now been corrected.

\section{References}

Arttamangkul S, Heinz DA, Bunzow JR, Song X, and Williams JT (2018) Cellular tolerance at the $\mu$-opioid receptor is phosphorylation dependent. eLife 7:e34989.

Bahouth SW and Nooh MM (2017) Barcoding of GPCR trafficking and signaling through the various trafficking roadmaps by compartmentalized signaling networks. Cell Signal 36:42-55.

Bonacci TM, Mathews JL, Yuan C, Lehmann DM, Malik S, Wu D, Font JL, Bidlack JM, and Smrcka AV (2006) Differential targeting of Gbetagamma-subunit signaling with small molecules. Science 312:443-446.

Bowman SL and Puthenveedu MA (2015) Postendocytic sorting of adrenergic and opioid receptors: new mechanisms and functions. Prog Mol Biol Transl Sci 132: 189-206.

Bowman SL, Shiwarski DJ, and Puthenveedu MA (2016) Distinct G protein-coupled receptor recycling pathways allow spatial control of downstream $\mathrm{G}$ protein signaling. J Cell Biol 214:797-806.
Bowman SL, Soohoo AL, Shiwarski DJ, Schulz S, Pradhan AA, and Puthenveedu MA (2015) Cell-autonomous regulation of $\mathrm{Mu}$-opioid receptor recycling by substance $\mathrm{P}$. Cell Rep 10:1925-1936.

Broadbent D, Ahmadzai MM, Kammala AK, Yang C, Occhiuto C, Das R, and Subramanian H (2017) Roles of NHERF family of PDZ-binding proteins in regulating GPCR functions. Adv Immunol 136:353-385.

Caengprasath N and Hanyaloglu AC (2019) Hardwiring wire-less networks: spatially encoded GPCR signaling in endocrine systems. Curr Opin Cell Biol 57:77-82.

Calebiro D, Nikolaev VO, Gagliani MC, de Filippis T, Dees C, Tacchetti C, Persani L, and Lohse MJ (2009) Persistent cAMP-signals triggered by internalized G-proteincoupled receptors. PLoS Biol 7:e1000172.

Doll C, Konietzko J, Pöll F, Koch T, Höllt V, and Schulz S (2011) Agonist-selective patterns of $\mu$-opioid receptor phosphorylation revealed by phosphosite-specific antibodies. Br J Pharmacol 164:298-307.

Dunn HA and Ferguson SS (2015) PDZ Protein regulation of G protein-coupled receptor trafficking and signaling pathways. Mol Pharmacol 88:624-639.

Eichel K and von Zastrow M (2018) Subcellular organization of GPCR signaling. Trends Pharmacol Sci 39:200-208.

Feng B, Li Z, and Wang JB (2011) Protein kinase C-mediated phosphorylation of the $\mu$-opioid receptor and its effects on receptor signaling. Mol Pharmacol 79:768-775.

Ferrandon S, Feinstein TN, Castro M, Wang B, Bouley R, Potts JT, Gardella TJ, and Vilardaga JP (2009) Sustained cyclic AMP production by parathyroid hormone receptor endocytosis. Nat Chem Biol 5:734-742.

Gondin AB, Halls ML, Canals M, and Briddon SJ (2019) GRK Mediates $\mu$-Opioid Receptor Plasma Membrane Reorganization. Front Mol Neurosci 12

Goubaeva F, Ghosh M, Malik S, Yang J, Hinkle PM, Griendling KK, Neubig RR, and Smrcka AV (2003) Stimulation of cellular signaling and G protein subunit dissociation by $\mathrm{G}$ protein betagamma subunit-binding peptides. J Biol Chem 278 : 19634-19641.

Halls ML, Yeatman HR, Nowell CJ, Thompson GL, Gondin AB, Civciristov S, Bunnett NW, Lambert NA, Poole DP, and Canals M (2016) Plasma membrane localization of the $\mu$-opioid receptor controls spatiotemporal signaling. $S c i$ Signal 9:ra16.

Irannejad R, Tomshine JC, Tomshine JR, Chevalier M, Mahoney JP, Steyaert J, Rasmussen SG, Sunahara RK, El-Samad H, Huang B, et al. (2013) Conformationa biosensors reveal GPCR signalling from endosomes. Nature 495:534-538.

Jensen DD, Lieu T, Halls ML, Veldhuis NA, Imlach WL, Mai QN, Poole DP, Quach T, Aurelio L, Conner J, et al. (2017) Neurokinin 1 receptor signaling in endosomes mediates sustained nociception and is a viable therapeutic target for prolonged pain relief. Sci Transl Med $\mathbf{9}$

Just S, Illing S, Trester-Zedlitz M, Lau EK, Kotowski SJ, Miess E, Mann A, Doll C, Trinidad JC, Burlingame AL, et al. (2012) Differentiation of Opioid Drug Effects by Hierarchical Multi-Site Phosphorylation. Mol Pharmacol 83 (3):633-639.

Kliewer A, Schmiedel F, Sianati S, Bailey A, Bateman JT, Levitt ES, Williams JT, Christie MJ, and Schulz S (2019) Phosphorylation-deficient G-protein-biased $\mu$-opioid receptors improve analgesia and diminish tolerance but worsen opioid side effects. Nat Commun 10:367.

Lau EK, Trester-Zedlitz M, Trinidad JC, Kotowski SJ, Krutchinsky AN, Burlingame AL, and von Zastrow M (2011) Quantitative encoding of the effect of a partial agonist on individual opioid receptors by multisite phosphorylation and threshold detection. Sci Signal 4:ra52.

Lefkowitz RJ, Pitcher J, Krueger K, and Daaka Y (1997) Mechanisms of B-adrenergic receptor desensitization. Advances in Pharmacology 42:416-420.

Lehmann DM, Seneviratne AMPB, and Smrcka AV (2008) Small molecule disruption of $\mathrm{G}$ protein $\beta \gamma$ subunit signaling inhibits neutrophil chemotaxis and inflammation. Mol Pharmacol 73:410-418.

Logan T, Bendor J, Toupin C, Thorn K, and Edwards RH (2017) $\alpha$-Synuclein promotes dilation of the exocytotic fusion pore. Nat Neurosci 20:681-689.

Marchese A, Paing MM, Temple BRS, and Trejo J (2008) G protein-coupled receptor sorting to endosomes and lysosomes. Annu Rev Pharmacol Toxicol 48:601-629.

Miess E, Gondin AB, Yousuf A, Steinborn R, Mösslein N, Yang Y, Goldner M, Ruland JG, Bunemann M, Krasel C, et al. (2018) Multisite phosphorylation is required for sustained interaction with GRKs and arrestins during rapid $\mu$-opioid receptor desensitization. Sci Signal 11.

Moulédous L, Froment C, Burlet-Schiltz O, Schulz S, and Mollereau C (2015) Phosphoproteomic analysis of the mouse brain mu-opioid (MOP) receptor. FEBS Lett 589:2401-2408.

Pierce KL, Premont RT, and Lefkowitz RJ (2002) Seven-transmembrane receptors. Nat Rev Mol Cell Biol 3:639-650.

Puthenveedu MA, Lauffer B, Temkin P, Vistein R, Carlton P, Thorn K, Taunton J, Weiner OD, Parton RG, and von Zastrow M (2010) Sequence-dependent sorting of recycling proteins by actin-stabilized endosomal microdomains. Cell 143:761-773. Roman-Vendrell C, Yu YJ, and Yudowski GA (2012) Fast modulation of $\mu$-opioid receptor (MOR) recycling is mediated by receptor agonists. $J$ Biol Chem 287: $14782-14791$

Romero G, von Zastrow M, and Friedman PA (2011) Role of PDZ proteins in regulating trafficking, signaling, and function of GPCRs: means, motif, and opportunity. Adv Pharmacol 62:279-314.

Sankaranarayanan S, De Angelis D, Rothman JE, and Ryan TA (2000) The use of pHluorins for optical measurements of presynaptic activity. Biophys $J \mathbf{7 9}$ : 2199-2208.

Schindelin J, Arganda-Carreras I, Frise E, Kaynig V, Longair M, Pietzsch T, Preibisch S, Rueden C, Saalfeld S, Schmid B, et al. (2012) Fiji: an open-source platform for biological-image analysis. Nat Methods 9:676-682.

Soohoo AL and Puthenveedu MA (2013) Divergent modes for cargo-mediated control of clathrin-coated pit dynamics. Mol Biol Cell 24:1725-1734, S1-S12.

Sorkin A and von Zastrow M (2009) Endocytosis and signalling: intertwining molecular networks. Nat Rev Mol Cell Biol 10:609-622.

Sriram K and Insel PA (2018) G protein-coupled receptors as targets for approved drugs: how many targets and how many drugs? Mol Pharmacol 93:251-258. 
Surve CR, Lehmann D, and Smrcka AV (2014) A chemical biology approach demonstrates $\mathrm{G}$ protein $\beta \gamma$ subunits are sufficient to mediate directional neutrophil chemotaxis. J Biol Chem 289:17791-17801.

Tanowitz M and von Zastrow M (2003) A novel endocytic recycling signal that distinguishes the membrane trafficking of naturally occurring opioid receptors. J Biol Chem 278:45978-45986.

Temkin P, Lauffer B, Jäger S, Cimermancic P, Krogan NJ, and von Zastrow M (2011) SNX27 mediates retromer tubule entry and endosome-to-plasma membrane trafficking of signalling receptors. Nat Cell Biol 13:715-721.

Thomsen ARB, Jensen DD, Hicks GA, and Bunnett NW (2018) Therapeutic targeting of endosomal G-protein-coupled receptors. Trends Pharmacol Sci 39: 879-891.

Tsvetanova NG and von Zastrow M (2014) Spatial encoding of cyclic AMP signaling specificity by GPCR endocytosis. Nat Chem Biol 10:1061-1065.

Vilardaga JP, Jean-Alphonse FG, and Gardella TJ (2014) Endosomal generation of cAMP in GPCR signaling. Nat Chem Biol 10:700-706.

Vistein R and Puthenveedu MA (2013) Reprogramming of G protein-coupled receptor recycling and signaling by a kinase switch. Proc Natl Acad Sci USA 110 15289-15294.

Wang F, Chen X, Zhang X, and Ma L (2008) Phosphorylation state of mu-opioid receptor determines the alternative recycling of receptor via Rab4 or Rab11 pathway. Mol Endocrinol 22:1881-1892.

Weinberg ZY, Crilly SE, and Puthenveedu MA (2019) Spatial encoding of GPCR signaling in the nervous system. Curr Opin Cell Biol 57:83-89.
Weinberg ZY, Zajac AS, Phan T, Shiwarski DJ, and Puthenveedu MA (2017) Sequence-specific regulation of endocytic lifetimes modulates arrestin-mediated signaling at the $\mu$ opioid receptor. Mol Pharmacol 91:416-427.

Williams JT, Ingram SL, Henderson G, Chavkin C, von Zastrow M, Schulz S, Koch T, Evans CJ, and Christie MJ (2013) Regulation of $\mu$-opioid receptors: desensitization, phosphorylation, internalization, and tolerance. Pharmacol Rev 65:223-254.

Yarwood RE, Imlach WL, Lieu T, Veldhuis NA, Jensen DD, Klein Herenbrink C, Aurelio L, Cai Z, Christie MJ, Poole DP, et al. (2017) Endosomal signaling of the receptor for calcitonin gene-related peptide mediates pain transmission. Proc Natl Acad Sci USA 114:12309-12314.

Yousuf A, Miess E, Sianati S, Du YP, Schulz S, and Christie MJ (2015) Role of phosphorylation sites in desensitization of $\mu$-opioid receptor. Mol Pharmacol 88:825-835.

Yudowski GA, Puthenveedu MA, Henry AG, and von Zastrow M (2009) Cargomediated regulation of a rapid Rab4-dependent recycling pathway. Mol Biol Cell 20:2774-2784.

Yudowski GA, Puthenveedu MA, and von Zastrow M (2006) Distinct modes of regulated receptor insertion to the somatodendritic plasma membrane. Nat Neurosci 9:622-627.

Address correspondence to: Manojkumar A. Puthenveedu, Department of Pharmacology, University of Michigan, 3422 Medical Science Unit I, Ann Arbor, MI 48109. E-mail: puthenve@umich.edu 